\title{
Radiation Produces Irreversible Chronic Dysfunction in the Submandibular Glands of the Rat
}

\author{
De la Cal $\mathrm{C}_{2}^{1}$, Fernández-Solari $\mathrm{J}^{2}$, Mohn $\mathrm{CE}^{3}$, Prestifilippo $\mathrm{JP}^{4, *}$, Pugnaloni $\mathrm{A}^{2}$, Medina $\mathrm{VA}^{5}$ and \\ Elverdin $\mathrm{JC}^{2}$
}

\author{
${ }^{1}$ Oral Pediatric Department, School of Dentistry, University of Buenos Aires, Buenos Aires, Argentina \\ ${ }^{2}$ Physiology Department, School of Dentistry, University of Buenos Aires, Buenos Aires, Argentina and National Scien- \\ tific and Technical Research Council (CONICET, Argentina) \\ ${ }^{3}$ Pathology Department, School of Dentistry, University of Buenos Aires, Buenos Aires, Argentina and National Scien- \\ tific and Technical Research Council (CONICET, Argentina) \\ ${ }^{4}$ Physiopathology Department, School of Pharmacy and Biochemistry. University of Buenos Aires \\ ${ }^{5}$ Laboratory of Radioisotopes, School of Pharmacy and Biochemistry, University of Buenos Aires and National Scientific \\ and Technical Research Council (CONICET, Argentina)
}

\begin{abstract}
The exposure to high doses of ionizing radiation during radiotherapy results in severe morphological and functional alterations of the salivary glands, such as xerostomia. In the present study we investigated the chronic effect of a single radiation dose of 15 Gray (Gy) limited to head and neck on rat salivary gland function (salivary secretion and gland mass) and histology. Results indicate that norepinephrine (NE)-induced salivary secretion was reduced significantly at 30 , 90, 180 and 365 days after the administration of a single dose of 15 Gy of ionizing radiation compared to non-irradiated animals. The maximal secretory response was reduced by $33 \%$ at 30 and 90 days post irradiation. Interestingly, a new fall in the salivary response to NE was observed at 180 days and was maintained at 365 days post irradiation, showing a $75 \%$ reduction in the maximal response. The functional fall of the salivary secretion observed at 180 days post irradiation was not only associated with a reduction of gland mass but also to an alteration of the epithelial architecture exhibiting a changed proportion of ducts and acini, loss of eosinophilic secretor granular material, and glandular vacuolization and fibrosis. On the basis of the presented results, we conclude that ionizing radiation produces irreversible and progressive alterations of submandibular gland (SMG) function and morphology that leads to a severe salivary hypo-function.
\end{abstract}

Keywords: Radiotherapy, xerostomia, submandibular gland, salivary secretion.

\section{INTRODUCTION}

Radiotherapy is a central treatment modality administered for head and neck cancers. Despite the great improvement in the technology for delivering therapeutic radiation, after irradiation, salivary glands are unavoidably irradiated causing devastating side-effects including severe gland functional and structural alterations which result in salivary dysfunction and consequent irreversible xerostomia ("dry mouth"). Radiation produces several oral complications that can be particularly distressing for the patient and include dysphagia, mucositis, sialoadenitis, rampant dental caries, increased tooth decay, oral infections, oesophagitis and gustatory dysfunction [1-4]. Therefore, there is a continuous effort to better understand the damage mechanism involved in ionizing radiation-induced salivary gland dysfunction which may facilitate improvements in the treatment or a

\footnotetext{
*Address correspondence to this author at the Cátedra de Fisiología, Facultad de Odontología, Universidad de Buenos Aires, Marcelo T de Alvear 2142, piso 3, sector A Ciudad de Buenos Aires C1122, Argentina; Tel: +54-11-4964-1275; Fax: +54-11-4508-3958;

E-mail: jprestifilippo@yahoo.com.ar
}

preventive strategy. Although salivary glands should be considered to be radioresistant because of their highly differentiated cellular state, they exhibit an exquisite sensitivity to radiation [5].

Salivary glands consist of several cell types: acinar cells which are responsible for water and protein secretion, myoepithelial cells surrounding the acini and ducts, and ductal cells which mainly modulate the ionic composition of the saliva. Irreversible hyposalivation after irradiation-induced damage is caused not only by the death of acinar cells, but also by a destruction of primitive glandular stem cells with progenitor capability. There is also a marked decline in functional differentiation and proliferative capacity of all of the surviving cells, which prevents replenishment of glandular cells [4, 6-9].

The homeostasis of the salivary glands, as well as any tissue, requires a balance between cellular death and proliferation, which is altered by ionizing radiation. Ionizing radiation induces apoptosis and proliferation during the early post radiation phase in submandibular gland (SMG) acini and ducts of the mouse [10]. However, it was recently reported that the imbalance between apoptosis and prolifera- 
tion induced by radiation causes the impairment of the SMG during the late radiation damage in the mouse [11], and that the increased apoptosis is the main cause of the imbalance [5, 12-16]. Moreover, we recently demonstrated the presence of apoptotic acinar and ductal cells distributed randomly in the SMG 1, 4 and $12 \mathrm{~h}$ after radiation exposure [16].

In the present study we investigated the chronic effect of a single radiation dose of 15 Gy limited to head and neck on salivary gland function (salivary secretion and gland mass) and histology.

\section{MATERIAL AND METHODS}

\section{Animals}

Adult female Wistar rats (250-300 g) were housed in group cages under standard conditions of light and temperature (lights on from $6 \mathrm{AM}$ to $8 \mathrm{PM}$ and $22-24{ }^{\circ} \mathrm{C}$ ) and with free access to rat chow and tap water. The animals were divided into several experimental groups with 6-8 animals each and kept in the laboratory area. The experimental procedures reported here were in accordance with recommendations of the Guide for the Care and Use of Laboratory Animals of the National Research Council, USA, 1996.

\section{Irradiation}

Anaesthetized (Ketamine $10 \mathrm{mg} / \mathrm{kg}$ and Xylazine $8 \mathrm{mg} / \mathrm{Kg}$, i.m.) rats were irradiated to the head and neck region with a single non lethal dose of gamma ray radiation of 15 Gy using a gamma-ray apparatus (Theratron 780) with ${ }^{60}$ Cobalt bomb at a dose rate of approximately $1 \mathrm{~Gy} / \mathrm{min}$ with an $80 \mathrm{~cm}$ source to skin distance. This radiation dose induces in the rat SMG similar effects exerted in patients treated with radiotherapy. Animals were returned to their home cages following irradiation. Control animals were anaesthetized but not exposed to radiation. All irradiations were done between 10:00 and 12:00 a.m.

\section{Determination of Salivary Response}

Salivary responses were determined at $2 \mathrm{~h}$, and 30, 90, 180 and 365 days post radiation exposure in rats under anesthesia (chloralose $100 \mathrm{mg} / \mathrm{kg} 0.5 \mathrm{ml} \mathrm{NaCl}$ (0.9\%), i.v.). For untreated curves, non-irradiated rats of each group were used. The right femoral vein was cannulated with a polyethylene catheter (P40 catheter, Rivera \& Cia, Argentina) to administered the sialagogic agonist, norepinephrine (NE), used in the study. Through a midline incision in the neck the trachea was intubated and the SMG ducts were exposed and cannulated with a fine glass cannula to collect saliva samples. No basal salivation was observed from the glands. Salivation was induced by the administration of different concentrations of NE ranging from the threshold dose to the dose that exerts the maximum stimulus $(0.3,1,3,10$ and 30 $\mu \mathrm{g} / \mathrm{kg}$, in saline) (Sigma Chemical Co., St. Louis, MO, USA) sequentially injected via the right femoral vein. Salivary samples were collected for 3 minutes in pre-weighed aluminum foil and the quantity of saliva was determined by weighing, as previously described. Three additional minutes were allowed until the administration of the next dose. Results were expressed as mg of saliva/gland.

\section{Body and Glandular Weight Measurement}

The body weight was measured at $2 \mathrm{~h}$, and 30, 90, 180 and 365 days post radiation exposure. Once the rats were anesthetized with ether, the SMG were dissected and weighed (wet glandular weight). Finally, the SMG were put in a dry stove during $12 \mathrm{~h}$ at $180{ }^{\circ} \mathrm{C}$ and then weighed (dry glandular weight).

\section{Histopathological Studies}

Salivary glands were removed and were fixed with $10 \%$ neutral buffered formalin. Tissue samples were embedded in paraffin and cut into serial sections of $5 \mu \mathrm{m}$ thick. SMG morphology and histopathological characteristics were examined on tissue sections after hematoxylin-eosin staining. Mucous and serous acini and intercalated and striated ducts were counted in rats after 180 days post radiation and were expressed by number of elements per 100x-magnification field.

\section{Statistics}

Data are presented as the means \pm SEM. For comparisons between 2 independent groups a Student's t-test was performed. For comparisons between different groups, a oneway analysis of variance (ANOVA) was performed followed by the Newman Keuls's multiple comparison test. All statistical analyses were performed with GraphPad Prism Version 5.00 software (San Diego, CA, USA). Differences with $p$ values $<0.05$ were considered statistically significant.

\section{RESULTS}

\section{Chronic Effect of Ionizing Radiation on Salivary Secretion}

In order to study the effect of ionizing radiation on rat stimulated salivary secretion we administered different doses of NE to non-irradiated and irradiated animals. Results indicate that salivary secretion was significantly reduced in rats at all days post irradiation studied. The maximal response to $\mathrm{NE}$ was diminished by $33 \%$ in 30 days post irradiated rats in comparison to that of non-irradiated rats. The percentage of diminution was maintained at 90 days post radiation exposure and was not significantly different from the salivation reduction observed after $2 \mathrm{~h}$ post irradiation (Fig. 1A, B, E). Interestingly, after 180 days post irradiation a new fall in the salivary secretion was observed showing a $75 \%$ reduction in the maximal response to NE in comparison to non-irradiated animals. This decrease was maintained in 365 days post irradiated rats (Fig. 1C, D, E).

We did not observe significant differences between the groups of non-irradiated rats regarding the dose response curves, suggesting that presented results are age-independent (Fig. 1F).

\section{Chronic Effect of Radiation on Body and Glandular Weights}

To determine whether the salivary secretion diminution was associated with a reduction in gland mass we evaluated the body and glandular weights in all animal groups. A reduction of about $12 \%$ was observed in the body weight of 
A

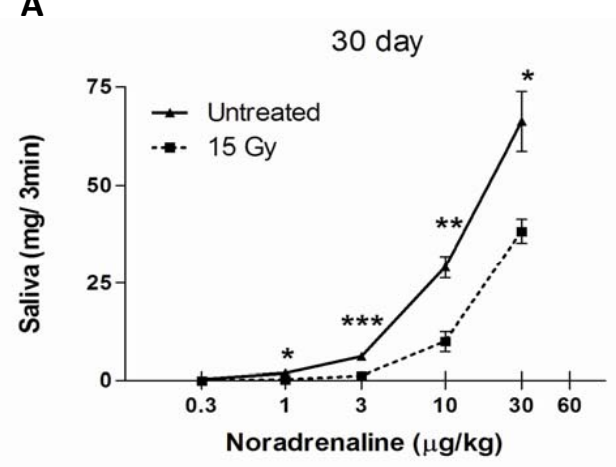

C

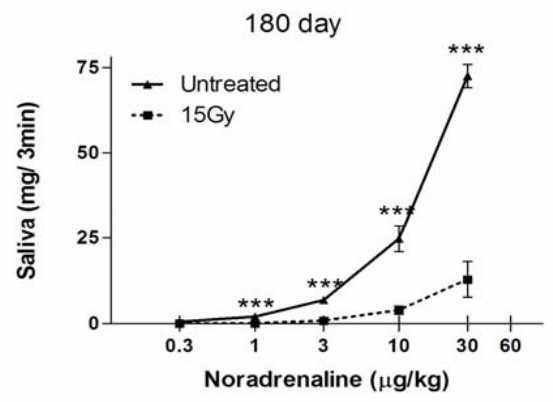

E

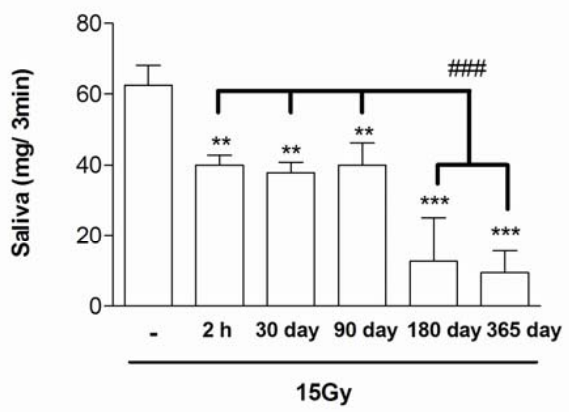

B

B 90 day

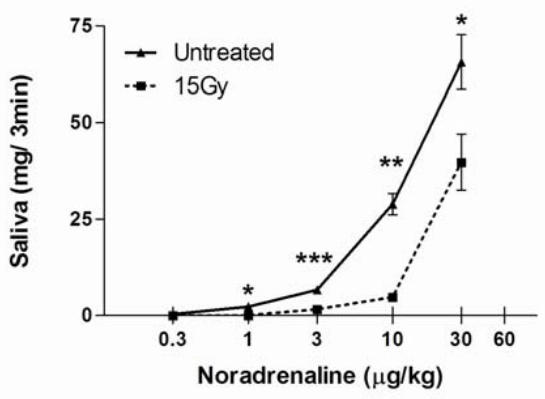

D 365 day

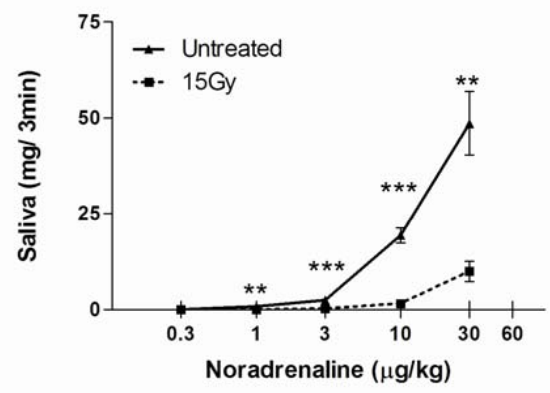

$\mathbf{F}$

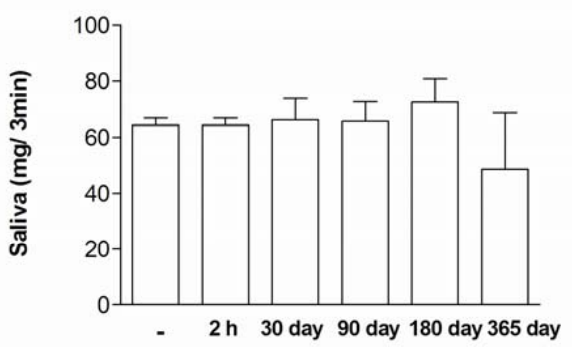

Fig. (1). Salivary secretion induced by NE in non-irradiated and 30 (A), 90 (B), 180 (C) and 365 (D) days post irradiated rats. Values represent means + SEM of 6-8 animals per group. Maximal secretory response obtained to NE in irradiated (E) and non-irradiated (F) rats. Values represent means \pm SEM of 6-8 animals per group. $* \mathrm{p}<0.05, * * \mathrm{p}<0.01$ and $* * * \mathrm{p}<0.001$ versus respective control. \#\#\#p<0.001 versus $2 \mathrm{~h}, 30$ and 90 days post irradiation.

Table 1. SMG and Body Weight in Irradiated Animals

\begin{tabular}{|c|c|c|c|c|c|c|}
\hline & Control & $\mathbf{2}$ hours & $\mathbf{3 0}$ days & $\mathbf{9 0}$ days & $\mathbf{1 8 0}$ days & 365 days \\
\hline \hline Body weight $(\mathrm{g})$ & $302.33 \pm 9.55$ & $290.82 \pm 3.53$ & $268.03 \pm 20.35$ & $266.17 \pm 9.66$ & $262.54 \pm 10.93$ & $234.75 \pm 3.71$ \\
\hline Glandular wet weight $(\mathrm{mg})$ & $258.71 \pm 11.12$ & $239.89 \pm 14.51$ & $214.49 \pm 22.32$ & $199.39 \pm 9.28$ & $188.72 \pm 16.52 *$ & $139.21 \pm 8.62 * *$ \\
\hline Glandular dry weight $(\mathrm{mg})$ & $54.27 \pm 3.36$ & $58.43 \pm 3.11$ & $46.64 \pm 5.94$ & $40.8 \pm 1.52 *$ & $39.55 \pm 3.64 *$ & $30.87 \pm 3.04 * * *$ \\
\hline \% SMG wet weight/ total body weight & $0.086 \pm 0.004$ & $0.083 \pm 0.005$ & $0.080 \pm 0.008$ & $0.075 \pm 0.004$ & $0.072 \pm 0.006 *$ & $0.059 \pm 0.004 *$ \\
\hline
\end{tabular}

Body, wet and dry glandular weight measured in $2 \mathrm{~h}$ and 30, 90, 180 and 365 days post irradiated rats. SMG's percentage of body weight (SMG weights were divided by total body weight in grams and multiplied by 100 ). Values represent means \pm SEM of 6-8 animals per group. $* \mathrm{p}<0.05$, **p $<0.01$ and $* * * \mathrm{p}<0.001$ versus respective control.

rats after 30, 90 and 180 days post irradiation in comparison to non-irradiated animals. A further decrease was demonstrated in irradiated animals after 365 days (22\%) (Table 1).

Regarding the glandular weight, we showed proportional changes in both wet and dry weights, showing 23-25\%, 27 $\%$ and $43-49 \%$ reduction in 90,180 and 365 days post irra- diated rats, respectively. No significant differences were observed in either body or glandular weight in $2 \mathrm{~h}$ post irradiated animals (Table $\mathbf{1}$ ).

In order to better understand the effect of radiation on glandular mass we compared the SMG wet weight as a percentage of body weight in irradiated and non-irradiated rats. 
A

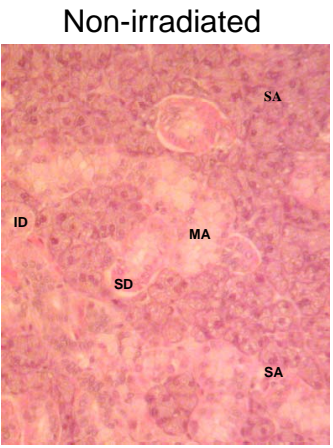

B

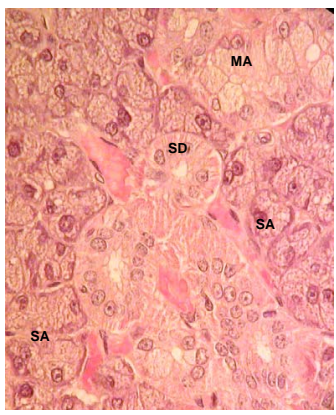

Fig. (2). Histological appearance of non-irradiated and 15 Gy dose irradiated SMG at 180 days. (A) 100x magnification. (B) 400x magnification. Scale bar $20 \mu \mathrm{m}$. MC: mucous acinos. SA: serous acinos. SD: striated duct. ID: intercalary duct. SD: striated duct. F: fibrosis. V: vacuoles.

A significant reduction of SMG wet weight relative to body weight was observed in irradiated animals after 180 and 365 days post irradiation (Table 1).

\section{Effect of Ionizing Radiation on SMG Morphology}

SMG salivary secretion showed a functional break at 180 days post irradiation, so we evaluated the histology of the gland at the same time. Ionizing radiation produced an alteration of the epithelial architecture exhibiting a changed proportion of ducts and acini, a considerable vacuolization of glandular cells, partial loss of eosinophilic secretor granular material, and glandular fibrosis (Fig. 2)

In addition, the number of serous and mucous acini per field was significantly reduced in SMG derived from 180 days post irradiated in comparison to non-irradiated animals (Fig. 3A). However, we did not detect significant variation in the number of intercalated ducts per field between SMG of irradiated and non-irradiated rats. Although we showed a significant increase in the number of striated ducts per field at 180 days post irradiation, this could be due to the diminution in the number of acini that facilitated the striated ducts over-expression (Fig. 3B).

\section{DISCUSSION}

Radiotherapy is the treatment employed commonly for head and neck tumors. It is usually observed that radiation therapy leads to severe dysfunction of the salivary glands followed by xerostomia because these tissues are highly sensitive to radiation [1-3]. This pathological condition in humans is manifested by oral dryness, impairment of normal oral functions (speech, chewing, and swallowing) because of insufficient wetting, and decreased lubrication of the mu-

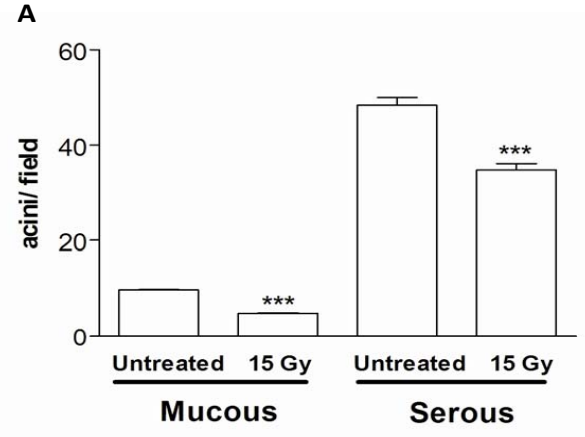

B

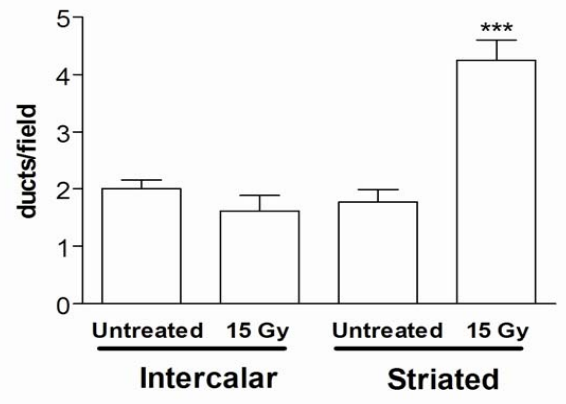

Fig. (3). Number of mucous and serous acini per field (A) and number of intercalated and striated ducts per field $(\mathbf{B})$ in SMG of 180 days post irradiated rats. Values represent means \pm SEM of 6-8 animals per group. $* * * \mathrm{p}<0.01$ versus respective control.
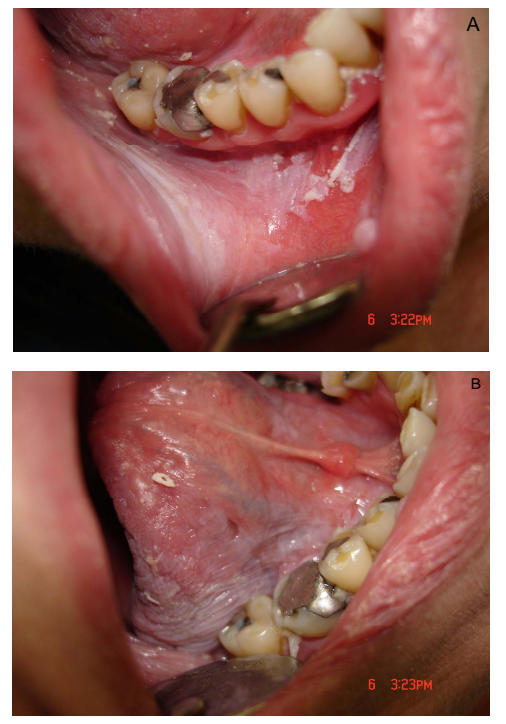

Fig. (4). Photographic view of the lesion in the mouth after ionizing radiation in humans showing mucositis of the gum and cheek, and incipient atrophy and desquamation of the oral mucosa (A), and dry mouth accompanied by ulceration and mucositis of the tongue $(\mathbf{B})$.

cosal surfaces and of ingested food. Furthermore, the oral mucosa can become dry and atrophic, leading to frequent ulceration and injury (Fig. 4A and B). Finally, the shift in the oral microflora towards cariogenic bacteria, the reduced salivary flow (oral clearance), and changes in saliva composition (decreased buffer capacity, $\mathrm{pH}$, immunoprotein concentrations) may result in rapidly progressing radiation caries (2, 5). 
The results of the present study demonstrated that a single non-lethal dose of head radiation in rats causes a chronic irreversible decrease in salivary secretion. This observation is in agreement with previous publications where radiation induced irreversible changes in salivary gland secretion [4, 17].

Radiation decreased the salivary secretion at all times studied post irradiation. The secretory activity of the gland did not vary significantly between 30 and 90 days post irradiation when a $33 \%$ reduction was observed of the maximal response obtained to NE compared to control rats. In addition, these dose response curves are very similar to that obtained at $2 \mathrm{~h}$ post irradiation in agreement with the results previously reported by our group [16]. Moreover, we observed a new fall in the salivary secretion at 180 days post radiation exposure that shows a $75 \%$ reduction in the maximal secretory response in comparison to that of nonirradiated rats. This reduction is maintained at 365 days post irradiation. The functional break observed at 180 days post irradiation is associated with a significant diminution in the wet and dry glandular weights relative to body weight. Therefore, the chronic secretory hypo-function observed could be not only due to alterations in the functional capability but also to progressive tissue damage that leads to a reduction of glandular mass. In this light, it was reported that late damage to the DNA leads to a diminution of mitotic velocity. This takes place at 90 days post irradiation [4].

Furthermore, we have recently reported the increase of apoptotic cells in the SMG just an hour after the exposure to a $15 \mathrm{~Gy}$ dose of gamma radiation and also a higher increase at $12 \mathrm{~h}$ after radiation exposure [16]. At this time, we also observed a decline in SMG cell proliferation evaluated by the incorporation of the timidine analog, bromo-2'deoxyuridine (data not shown). These results, in agreement with other authors, reveal that radiation causes an imbalance between apoptotic and proliferative mechanisms, with the former a primary mechanism of injury induced in the salivary glands during radiotherapy [5, 12-16]. It was also reported that after 120-240 days post irradiation the expression of damage due to the salivary gland dysfunction can result from the death of stem cells [18].

On the other hand, it was reported that a 20 Gy radiation dose did not affect the NE content in the salivary glands indicating that the diminution in the secretory responses does not correspond to alterations in the autonomic efferent nerves [9].

Consequently, the ionizing radiation produced the loss of reparatory capability of the tissue that leads progressively to the atrophy of the gland. Additionally, we showed a time dependent diminution in the corporal weight of irradiated rats that is probably due to the lack of saliva that leads to an inadequate oral digestion.

Since it was reported a time dependent progressive loss of salivary function in humans [19], we also measured the salivary secretion in non-irradiated rats at different ages. Results indicate that no significant diminution in the salivary secretion of non-irradiated rats at different ages was observed suggesting that presented results are age-independent.
The functional fall of the salivary secretion observed at 180 days post irradiation was not only associated with a reduction of gland mass but also to an alteration of the epithelial architecture exhibiting a changed proportion of ducts and acini, loss of eosinophilic secretor granular material, and glandular vacuolization and fibrosis.

\section{CONCLUSION}

On the basis of the presented results, we conclude that ionizing radiation produces irreversible and progressive alterations of submandibular gland function and morphology that leads to a severe salivary hypo-function.

\section{ACKNOWLEDGEMENTS}

This work was supported by grants from the University of Buenos Aires (UBACyT O007) and 20020090300039. Fundación Alberto J. Roemmers and National Agency of Scientific and Technological Promotion (PICT-01016). The authors are greatly indebted to Ricardo Horacio Orzuza for technical assistance.

\section{CONFLICT OF INTEREST}

None declared.

\section{REFERENCES}

[1] Dreizen S, Daly TE, Drane JB, Brown LR. Oral complications of cancer radiotherapy. Postgrad Med 1977; 61: 85-92.

[2] Franzén L, Funegård U, Ericson T, Henriksson R. Parotid gland function during and following radiotherapy of malignancies in the head and neck. A consecutive study of salivary flow and patient discomfort. Eur J Cancer 1992; 28: 457-62.

[3] Valdez IH, Atkinson JC, Ship JA, Fox PC. Major salivary gland function in patients with radiation-induced xerostomia: flow rates and sialochemistry, Int J Radiat Oncol Biol Phys 1993; 25: 41-7.

[4] Nagler RM, Baum BJ, Miller G, Fox PC. Long-term salivary effects of single-dose head and neck irradiation in the rat. Arch Oral Biol 1998; 43: 297-303.

[5] Nagler RM. The enigmatic mechanism of irradiation-induced damage to the major salivary glands. Oral Dis 2002; 8:141-6.

[6] Zeilstra LJ, Vissink A, Konings AW, Coppes RP. Radiation induced cell loss in rat submandibular gland and its relation to gland function. Int J Radiat Biol 2000; 76: 419-29.

[7] Garrett JR, Kidd A. The innervation of salivary glands as revealed by morphological methods. Microsc Res Tech 1993; 26: 75-91.

[8] Nagler RM, Hershkovich O. Age-related changes in unstimulated salivary function and composition and its relations to medications and oral sensorial complaints. Aging Clin Exp Res 2005; 17: 35866.

[9] Kohn WG, Grossman E, Fox PC, Armando I, Goldstein DS, Baum BJ. Effect of ionizing radiation on sympathetic nerve function in rat parotid glands. J Oral Pathol Med 1992; 21: 134-7.

[10] Bralic M, Muhvic-Urek M, Stemberga V, et al. Cell death and cell proliferation in mouse submandibular gland during early postirradiation phase. Acta Med Okayama 2005; 59: 153-9.

[11] Muhvic-Urek M, Bralic M, Curic S, Pezelj-Ribaric S, Borcic J, Tomac J. Imbalance between apoptosis and proliferation causes late radiation damage of salivary gland in mouse. Physiol Res 2006; 55: 89-95.

[12] Hall EJ, Giaccia AJ. In: Hall EJ, Giaccia AJ, Eds. Clinical response of normal tissues. Radiobiology for radiobiologists. Philadelphia, Lippincott Williams and Wilkins 2006; 327-48.

[13] Avila JL, Grundmann O, Burd R, Limesand KH. Radiation-induced salivary gland dysfunction results from p53-dependent apoptosis. Int J Radiat Oncol Biol Phys 2009; 73: pp. 523-9.

[14] Limesand KH, Schwertfeger KL, Anderson SM. MDM2 is required for suppression of apoptosis by activated Akt1 in salivary acinar cells. Mol Cell Biol 2006; 26: 8840-56.

[15] Lee HJ, Lee YJ, Kwon HC, et al. Radioprotective effect of heat shock protein 25 on submandibular glands of rats. Am J Pathol 2006; 169:1601-11. 
[16] de la Cal C, Lomniczi A, Mohn CE,et al. Decrease in salivary secretion by radiation mediated by nitric oxide and prostaglandins. Neuroimmunomodulation 2006; 13: 19-27.

[17] O'Connell AC, Redman RS, Evans RL, Ambudkar IS. Radiationinduced progressive decrease in fluid secretion in rat submandibular glands is related to decreased acinar volume and not impaired calcium signaling. Radiat Res 1999; 151:150-8.
[18] Konings AW, Faber H, Vissink A, Coppes RP. Radioprotective effect of amifostine on parotid gland functioning is region dependent. Int J Radiat Oncol Biol Phys 2005; 63:1584-91.

[19] Nagler RM, Hershkovich O. Age-related changes in unstimulated salivary function and composition and its relations to medications and oral sensorial complaints. Aging Clin Exp Res 2005; 17: 35866.

(C) Cal C et al.; Licensee Bentham Open.

This is an open access article licensed under the terms of the Creative Commons Attribution Non-Commercial License (http://creativecommons.org/licenses/by-nc/3.0/) which permits unrestricted, non-commercial use, distribution and reproduction in any medium, provided the work is properly cited. 